\title{
Myelin-forming cell-specific cadherin-19 is a marker for minimally infiltrative glioblastoma stem-like cells
}

\author{
Michael Zorniak, PhD, ${ }^{1,2}$ Paul A. Clark, PhD, ${ }^{2}$ and John S. Kuo, MD, PhD'-5 \\ ${ }^{1}$ Neuroscience Training Program, Departments of ${ }^{2}$ Neurological Surgery and ${ }^{3}$ Human Oncology, ${ }^{4}$ Carbone Cancer Center, and \\ ${ }^{5}$ Center for Stem Cell and Regenerative Medicine, University of Wisconsin School of Medicine and Public Health, Madison, \\ Wisconsin
}

OBJECT Glioblastoma stem-like cells (GSCs) exhibit stem-like properties, are highly efficient at forming tumor xenografts, and are resistant to many current therapies. Current molecular identifiers of GSCs are scarce and controversial. The authors describe differential cell-surface gene expression profiling to identify GSC-specific markers.

METHODS Independent human GSC lines were isolated and maintained in standard neural stem cell (NSC) media and were validated for self-renewal, multipotent differentiation, and tumor initiation properties. Candidate upregulated GSCspecific plasma membrane markers were identified through differential Affymetrix U133 Plus 2.0 Array gene expression profiling of GSCs, human NSCs (hNSCs), normal brain tissue, and primary/recurrent glioblastoma multiforme samples. Results were validated by using comparative quantitative reverse transcription polymerase chain reaction and Western blot analysis of GSCs, hNSCs, normal human astrocytes, U87 glioma cell line, and patient-matched serum-cultured glioblastoma multiforme samples.

RESULTS A candidate GSC-specific signature of 19 upregulated known and novel plasma membrane-associated genes was identified. Preferential upregulation of these plasma membrane-linked genes was validated by quantitative polymerase chain reaction. Cadherin-19 (CDH19) protein expression was enhanced in minimally infiltrative GSC lines.

CONCLUSIONS Gene expression profiling of GSCs has shown CDH19 to be an exciting new target for drug development and study of GBM tumorigenesis.

http://thejns.org/doi/abs/10.3171/2014.9.JNS132373

KEY WORDS cadherin-19; CDH19; gene expression profiling; glioblastoma multiforme; glioblastoma stem-like cells; oncology

$\mathrm{G}$ LIOBLASTOMa multiforme (GBM) is a highly malignant brain tumor for which the median survival is 14.6 months..$^{26} \mathrm{GBM}$ often recurs despite maximal tumor resection, radiotherapy, and chemotherapy. Glioblastoma stem-like cells (GSCs) have been hypothesized to initiate tumor recurrence and are resistant to current therapeutic approaches. . $^{2,3,7,16}$ Successful GBM therapies will need to address this recalcitrant tumor-initiating population of cells in combination with current strategies. ${ }^{10}$

To date, isolation or enrichment of cancer stem-like cells has mainly involved strategies derived from normal stem cell biology. In hematopoietic,,${ }^{4,21}$ breast, ${ }^{1}$ and brain malignancies, ${ }^{23-25}$ normal stem cell markers were used to first identify stem-like cancer cells, which were validated by their recapitulation of parental tumor pathology after serial implantation into immunodeficient mice. These approaches were effective in enriching for stem-like cancer cells; however, recent investigators have reported that some of the unlabeled cell populations also retain efficient tumor-initiating properties. ${ }^{5,19}$ Moreover, the current markers cannot safely serve as drug targets because they are also expressed by normal adult self-renewing stem cells.

ABBREVIATIONS ACTB = $\beta$-actin; $\mathrm{CDH}=$ cadherin; CNP = 2', 3'-cyclic-nucleotide 3'-phosphodiesterase; GBM = glioblastoma multiforme; GPC3 = glypican-3; GPR17 = $\mathrm{G}$ protein-coupled receptor 17; GSC = glioblastoma stem-like cell; HLA = human leukocyte antigen; hNSC = human neural stem cell; MCPIP = monocyte chemotactic protein 1-induced protein; MHC = major histocompatibility complex; NCBI GEO = National Center for Biotechnology Information Gene Expression Omnibus; NCI REMBRANDT = National Cancer Institute Repository of Molecular Brain Neoplasia Database; NHA = normal human astrocyte; OPC = oligodendrocyte progenitor cell; qRT-PCR = quantitative reverse transcription polymerase chain reaction.

SUBMITTED October 28, 2013. ACCEPTED September 18, 2014.

INCLUDE WHEN CITING Published online October 31, 2014; DOI: 10.3171/2014.9.JNS132373.

DISCLOSURE The authors report no conflict of interest concerning the materials or methods used in this study or the findings specified in this paper. 
We used an unbiased gene expression profiling-based approach to identify novel GSC-specific plasma membrane markers. Two GSC lines were characterized using gene microarrays compared with human neural stem cells (hNSCs), normal human brain, primary GBM, and recurrent GBM tissue samples. After filtering for plasma membrane transcripts, 19 GSC transcripts with multiple probe sets were found upregulated in contrast to findings in normal controls and whole GBM tumor samples. Candidate genes were validated by quantitative reverse transcription polymerase chain reaction (qRT-PCR) with two additional GSC lines along with normal human astrocytes (NHAs), the U87 glioma cell line, and the serum-cultured, patient-matched GBM lines 22T and 33T. Expression of cadherin-19 (CDH19) is restricted to minimally infiltrative GSCs, with no detectable protein in other GSCs, GBM cell lines, or normal neural cell lines on immunoblotting. These findings suggest that $\mathrm{CDH} 19$ (a Type II atypical cadherin specific to myelinating cells during development) ${ }^{31}$ could serve as a feasible marker for GSC identification, isolation, and drug discovery.

\section{Methods}

\section{GSC, GBM, and Control Cell Line Culture}

All studies were performed with approval from the University of Wisconsin-Madison Institutional Review Board with informed consent obtained from patients, and with approval from the Institutional Animal Care and Use Committee. Glioblastoma stem-like cells were isolated by following previously reported protocols, ${ }^{7,12,14,23,27}$ without the use of surface markers. Briefly, after the histological diagnosis was established based on WHO criteria, fresh GBM tissue was directly collected according to institutional review board-approved protocol, weighed, coarsely minced with a scalpel blade, and subsequently chopped twice at $200 \mu \mathrm{m}$ using a tissue chopper (Sorvall Tissue Chopper, model TC-2, Diversified Equipment). Chopped tissue was directly plated in a suspension and cultured in the following passaging medium: 70\% DMEM-high glucose, 30\% Ham's F12, 1× B27 supplement, $5 \mu \mathrm{g} / \mathrm{ml}$ heparin, and penicillin-streptomycin-amphotericin, supplemented with $20 \mathrm{ng} / \mathrm{ml}$ each of human recombinant epidermal growth factor and bovine fibroblast growth factor. ${ }^{27}$ Sphere cultures were passaged approximately every 7 days by tissue chopping twice at $100 \mu \mathrm{m}$. Individual patient-derived GSC lines 12.1, 22, 33, and 44 were cultured in suspension, and they were rigorously validated for self-renewal by neurosphere formation, expression of stem cell markers (i.e., $A C / C D 133$ ), multipotency, tumor initiation, and serial implantation in nonobese diabetic/severe combined immunodeficient (also called NOD-SCID) mice (Harlan Sprague-Dawley). ${ }^{8,30}$ Standard serum conditions were used to maintain patient-matched $22 \mathrm{~T}$ and $33 \mathrm{~T}$ GBM bulk tumor lines and U87 and normal human astrocyte (NHA) lines (DMEM, 10\% fetal bovine serum, 1\% antibiotics; Invitrogen). GSCs were compared with $\mathrm{hN}-$ SCs and were maintained as previously described..$^{27}$ Establishment and cryopreservation of cell cultures ranged from Passages 1 to 10. Cells used for experiments ranged from Passages 20 to 25 .

\section{Gene Expression Profiling}

Pooled gene expression profiling of human GSC lines 12.1 and $22(n=2)$ (National Center for Biotechnology Information Gene Expression Omnibus [NCBI GEO] accession nos. GSM1253303 and GSM1253304, respectively) were compared with hNSCs M031 CTX $(n=2)(\mathrm{NCBI}$ GEO accession nos. GSM458064 and GSM458065), normal human brain tissue $(n=21)$, primary GBM tumors $(n=21)$, and recurrent GBM tumors $(n=22)$ (Table 1). Total RNA was extracted from GSCs with an RNeasy Kit (Qiagen), and samples were then sent to LC Sciences for final gene expression processing and analysis. In brief, total RNA was reverse transcribed with T7-Oligo(dT) primers. After cleanup of cDNA, cRNA was synthesized using in vitro transcription ( $3^{\prime}$ IVT). cRNA was biotinylated and fragmented before hybridization to a U133 Plus 2.0 Array (Affymetrix) that contained probes for over 47,000 transcripts. Hybridized cRNA was labeled with streptavidin-phycoerythrin for visualization. Hybridization images were collected using a laser scanner (GenePix 4000B, Molecular Devices) and digitized using Array-Pro image analysis software (Media Cybernetics). U133 Plus 2.0 Array gene expression profiles for hNSCs were obtained from Dr. Clive Svendsen. Profiling data of gross tissue samples of normal human brain, primary GBM, and recurrent GBM were obtained from the National Cancer Institute Repository of Molecular Brain Neoplasia Database (NCI REMBRANDT) (Table 1). All of the expression profiles were batch-normalized by a robust multichip average algorithm using the Geospiza GeneSifter (PerkinElmer) online microarray database and analysis software. The data were then exported into Microsoft Office Excel (2010) and organized for GSC transcripts with raw intensity values 10-fold or higher over normal brain, hNSC, primary GBM, and recurrent GBM samples. The reverse-sorting algorithm was done to obtain downregulated GSC transcripts (NCBI GEO accession no. GSE51822). This analysis provided a list of upregulated plasma membrane transcripts in GSCs (Fig. 1). Since each transcript has multiple probes on U133 Plus 2.0 Arrays, all the probes for an upregulated transcript are shown to demonstrate signal redundancy. The data are represented as a heat map of raw intensity values with the provided scale.

\section{qRT-PCR Validation of mRNA Expression Profiles}

Gene expression profiling was validated by quantitative reverse transcription polymerase chain reaction (qRT-PCR) to confirm differential upregulation of GSC transcripts. RNA was isolated from the following cell lines using the RNeasy Kit: GSCs 12.1, 22, 33, and 44; hNSCs; NHAs; and serum cultured GBM tumor lines 22T and 33T. Isolated RNA was reverse transcribed with the Omniscript Reverse Transcription Kit (Qiagen) to create a cDNA pool for each cell line. A nonadeoxyribonucleotide random primer mixture (TaKaRa) and human placental optizyme ribonuclease inhibitor (Fisher Scientific) were used for reverse transcription per the manufacturer's instructions. Quantitative PCR was performed with the EXPRESS SYBR GreenER qPCR Supermix Universal Kit (Invitrogen). Reactions were scaled down to $20 \mu$, 
TABLE 1. Gene expression profile file names downloaded from NCI REMBRANDT

\begin{tabular}{clcc}
\hline No. & Normal Human Brain & GBM Primary & GBM Recurrent \\
\hline 1 & NT_HF0088_U133P2 & 1_E09233_U133P2 & 2_E09138_U133P2 \\
\hline 2 & NT_HF0120_U133P2 & 1_E09405_U133P2 & 2_E09139_U133P2 \\
\hline 3 & NT_HF0131_U133P2 & 1_E09451_U133P2 & 2_E09167_U133P2 \\
\hline 4 & NT_HF0137_U133P2 & 1_E09489_U133P2 & 2_E09192_U133P2 \\
\hline 5 & NT_HF0151_U133P2 & 1_E09511_U133P2 & 2_E09231_U133P2 \\
\hline 6 & NT_HF0163_U133P2 & 1_E09531_U133P2 & 2_E09483_U133P2 \\
\hline 8 & NT_HF0171_U133P2 & 1_E09654_U133P2 & 2_E09546_U133P2 \\
\hline 9 & NT_HF0178_U133P2 & 1_E09698_U133P2 & 2_E09601_U133P2 \\
\hline 10 & NT_HF0211_U133P2 & 1_E09704_U133P2 & 2_E09602_U133P2 \\
\hline 11 & NT_HF0232_U133P2 & 1_E09774_U133P2 & 2_E09606_U133P2 \\
\hline 12 & NT_HF0295_U133P2 & 1_E09782_U133P2 & 2_E09610_U133P2 \\
\hline 13 & NT_HF0303_U133P2 & 1_E09832_U133P2 & 2_E09624_U133P2 \\
\hline 14 & NT_HF0377_U133P2 & 1_E09846_U133P2 & 2_E09647_U133P2 \\
\hline 15 & NT_HF0383_U133P2 & 1_E09847_U133P2 & 2_E09649_U133P2 \\
\hline 16 & NT_HF0467_U133P2 & 1_E09857_U133P2 & 2_E09670_U133P2 \\
\hline 17 & NT_HF0512_U133P2 & 1_E09910_U133P2 & 2_E09787_U133P2 \\
\hline 18 & NT_HF0523_U133P2 & 1_E09917_U133P2 & 2_E09791_U133P2 \\
\hline 19 & NT_HF0526_U133P2 & 1_E09938_U133P2 & 2_E09802_U133P2 \\
\hline 20 & NT_HF0533_U133P2 & 1_E09964_U133P2 & 2_E09852_U133P2 \\
\hline 21 & NT_HF0593_U133P2 & 1_E09967_U133P2 & 2_E09868_U133P2 \\
\hline 22 & NT_HF0616_U133P2 & 1_E09998_U133P2 & 2_E09930_U133P2 \\
\hline & NA & NA & 2_E09965_U133P2 \\
\hline
\end{tabular}

$\mathrm{NA}=$ not applicable.

combined in an ABI Prism 96-well optical reaction plate, and loaded into an ABI Prism 7000 unit (Applied Biosystems). Primers were designed according to online tools at Integrated DNA Technologies (http://www.idtdna.com) and only chosen if the predicted amplicon contained 2 exon regions and a melting temperature near $60^{\circ} \mathrm{C}$ (Table 2). Intron spanning and gene specificity were confirmed by an NCBI BLAST search (http://blast.ncbi.nlm.nih. gov). $18 S r R N A$ was used as the reference gene. hNSC cycle threshold, or $\mathrm{C}(\mathrm{t})$, values were used as the calibrator for calculations. All other cell lines were set as the target. $C(t)$ values were chosen during linear growth of the produced sigmoidal curves. Gene expression was quantified by the $\Delta \Delta \mathrm{C}(\mathrm{t})$ method. In brief, the $\Delta \mathrm{C}(\mathrm{t})$ value was calculated by subtracting the $\mathrm{C}(\mathrm{t})$ of the target from the reference values. The $\Delta \Delta \mathrm{C}(\mathrm{t})$ was calculated by subtracting the $\Delta \mathrm{C}(\mathrm{t})$ of the target from the calibrator. Finally, the formula, $2^{\wedge}(-\Delta \Delta C(t))$, was used to report fold change differences over the calibrator value.

\section{Immunoblotting Analysis}

Immunoblotting was performed as described. ${ }^{30}$ Cells were lysed using cell extraction buffer (catalog no. FNN0011, Invitrogen) containing protease inhibitor cocktail (P8340, Sigma-Aldrich). Total protein was quantified using an EZQ Protein Quantification assay (R33200, Invitrogen). Thirty micrograms of protein was resuspended in $2 \times$ reducing sample buffer (LC2676, Invitrogen), electrophoresed on $10 \%-20 \%$ tris-glycine gels (Invitrogen), transferred using a semidry transfer system (Bio-Rad) to polyvinylidene difluoride membranes (Millipore), and probed with Ms-pAb-anti-CDH19 (H00028513-B01P, Abnova) at 1:250, identified at approximately $114 \mathrm{k} \mathrm{kDa}$. Rb-pAb-anti-ACTB (ab8227, abcam) at 1:2000 was used as a loading control. Immunocomplexes were detected using luminescence Supersignal West Pico Substrate (Thermo Scientific) per manufacturer's instructions.

\section{Results}

\section{Differential Plasma Membrane Transcripts in GSCs}

Gene expression profiles were sorted for differentially upregulated ( $>10$-fold) GSC plasma membrane transcripts compared with normal human (nontumor) brain, hNSCs, and primary GBM and recurrent GBM (Fig. 1A). Nineteen distinct transcripts with multiple redundant probe sets were found to be specifically upregulated in GSCs (Fig. 1B). As expected, PROM1 (i.e., CD133) expression in GSCs was not meaningfully upregulated (2.2-fold) compared with hNSCs, thus demonstrating the validity of our approach. Additionally, a set of downregulated GSC transcripts was generated that included reduced expression of 3 different human leukocyte antigen (HLA) genes (HLA-DRB1, HLA-DPAl, and HLA-DRA) in GSCs (Fig. 2).

\section{CDH19 Is Upregulated in Minimally Infiltrative GSCs}

qRT-PCR primers were designed for the signature of 

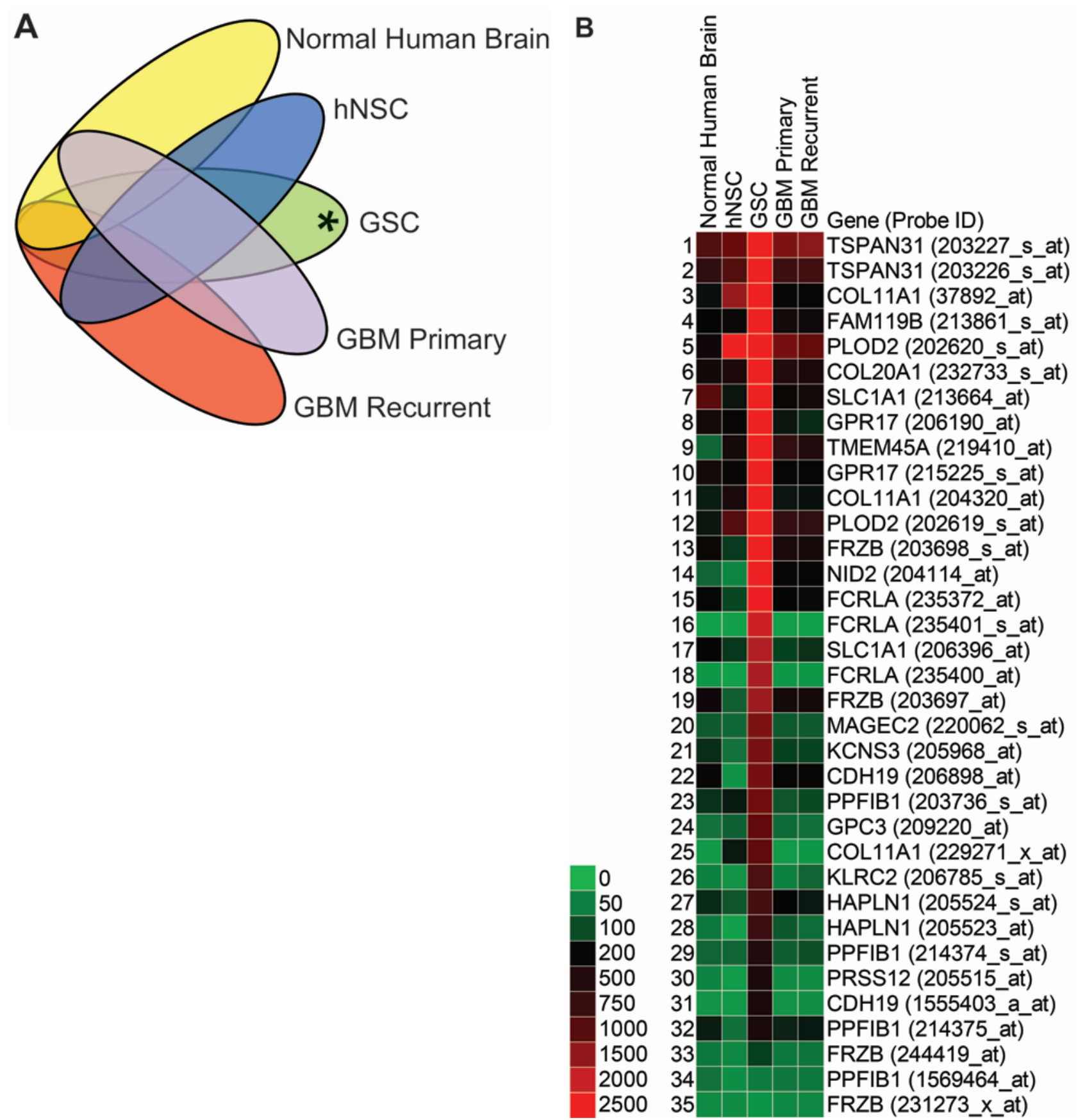

FIG. 1. Nineteen upregulated plasma membrane GSC-specific transcripts. A: Logic diagram showing how gene expression profiles were sorted to identify GSC-specific transcripts (asterisk). Normal human brain $(n=21)$, primary GBM $(n=21)$, and recurrent GBM $(n=22)$ gene expression profiles were obtained from NCI REMBRANDT (see Table 1). GSCs $(n=2)$ were isolated from two patients at the University of Wisconsin. hNSCs $(n=2)$ were isolated from two human fetal brains. B: Heat map with accompanying color key of raw intensity values from gene expression profiles. Data were filtered for plasma membrane transcripts and gated for GSC values greater than a 10 -fold change above all other samples. Acronyms correspond to official gene symbols found online at the NCBI. Multiple probe sets shown to demonstrate internal reproducibility.

19 upregulated GSC plasma membrane transcripts to validate the mRNA expression profiles (Table 2). Additional GSC lines 33 and 44 were added to this analysis along with serum-cultured NHAs, the U87 glioma cell line, and the patient-matched GBM lines 22T and 33T. GBM lines are representative of the non-stem-like cancer cell popu- lation. CDH19 demonstrated elevated transcript levels across all GSC samples compared with hNSCs, NHAs, the U87 cell line, and the GBM lines 22T and 33T (Fig. 3A). SLC1A1, TSPAN31, COL20A1, GPR17, and GPC3 also maintained similar expression profiles in qRT-PCR compared with the mRNA microarrays; however, their 
TABLE 2. qRT-PCR primers for gene expression profile validation

\begin{tabular}{lllrc}
\hline \multicolumn{1}{c}{ HGNC } & \multicolumn{1}{c}{ Forward $\left(5^{\prime}-\right.$ 3 $\left.^{\prime}\right)$} & \multicolumn{1}{c}{ Reverse $\left(5^{\prime}-3^{\prime}\right)$} & CDS & Exons \\
\hline 18S rRNA & GTTGGTGGAGCGATTTGTCTGGTT & TAGCATGCCAGAGTCTCGTTCGTT & $1345-1401$ & NA \\
\hline COL11A1 & AATGGAGCTGATGGACCACAAGGA & TCTCCAACACCACCAACTGAACCA & $3580-3641$ & $49-50$ \\
\hline COL20A1 & ACCTTGCAGATCTTCGAGCTCACT & TCCTCAATCACAAACTCCCTCCGA & $274-344$ & $4-5$ \\
\hline CDH19 & AGTCATCACATCGGCCAGCTAAGA & TACTTCCAGCTCCAGCTCCCAAA & $178-265$ & $2-3$ \\
\hline FAM119B & TGCTGACCATCACGCAGAACTTTG & CCTTCTTGCCTCGGAATCCACAT & $116-235$ & $1-2$ \\
\hline FCRLA & CAGCCACTGAGGACAACCAAGTTT & AAGCACCCTGCACTCTGATCTCTA & $776-841$ & $4-5$ \\
\hline FRZB & TGCCTCTGCCCTCCACTTAATGTT & TACCGAGTCGATCCTTCCACTTCT & $727-825$ & $4-5$ \\
\hline GPC3 & AACCAGCTCCTGAGAACCATGTCT & TCATCATCACCGCAGTCTCCACTT & $1408-1505$ & $6-7$ \\
\hline GPR17 & AAACGGAGTTGGTGGGCTGGAT & GCCACTTCAAGGCATTCATGCTT & $7-104$ & $2-3$ \\
\hline HAPLN1 & CTGTTGTGGTAGCACTGGACTTAC & CCCAGTCGTGGAAGTAAGGGAATAC & $446-503$ & $3-4$ \\
\hline KCNS3 & CCATGAAGTTGGGCTTCTGCTTCT & GGCTGGATGTGTGGTCATCTTTCT & $963-1060$ & NA \\
\hline KLRC2 & GCCAGCATTTTACCTTCCTCA & ACTGCACAGTTAAGTTCAGCAT & $493-623$ & $5-6$ \\
\hline MAGEC2 & TGCCAGACAGTGAGTCCTCTTTCA & ACAGGCTCCTCTGCTTCGTATTTG & $389-385$ & NA \\
\hline NID2 & GCCACAGCAGCATTGATGTTTCCT & TTGGTGTGAGGATCCAAGGTGGAA & $1013-1097$ & $4-5$ \\
\hline PLOD2 & TGGACCCACCAAGATTCTCCTGAA & AGTGCAGCCATTATCCTGTGTCCA & $765-840$ & $7-8$ \\
\hline PPFIBP1 & TGCCAGATCCCAGATTCAACAGCA & TCCCTGGTAGGTGTCCATTTGTCA & $214-292$ & $4-5$ \\
\hline PRSS12 & TTCTGGACTGGGCTTATTCCCAT & TTTCTTCATCTCCTCGGCAACGGA & $672-733$ & $2-3$ \\
\hline SLC1A1 & GAAGCAGTGGCAGCGGTTTTATT & ATGTGGCCGTGATACTGATGGTGA & $1120-1207$ & $10-11$ \\
\hline TMEM45A & TGGAGCTATTGCGGTCAAGTCTCA & TCCAATCTGAAAGAACCAGCTCCC & $536-594$ & $4-5$ \\
\hline TSPAN31 & TGCTGGTGAGCTTGTTGCTCATTG & ATGATGTGGATGCTGGACACCAGA & $62-137$ & $1-2$ \\
\hline
\end{tabular}

CDS = coding sequence; HGNC = Human Gene Nomenclature Committee-approved gene symbol.

upregulation did not uniformly extend to GSC lines 33 and 44. Western blot analysis confirmed 12.1 and 22 GSC-specific expression of CDH19 (Fig. 3B). These two GSC lines express oligodendrocyte and neural progenitor markers and were previously profiled as minimally infiltrative in xenograft studies..$^{30}$ The GSC lines 33 and 44 did not express $\mathrm{CDH} 19$ at the protein level (data not shown).

\section{CDH19 Expression in Normal Human Tissues and GBM Tumors}

In human tissues, $\mathrm{CDH} 19$ transcript (probe ID: 206898_at) was expressed meaningfully in only two tissues, olfactory bulb and dorsal root ganglion (Fig. 4), but their respective raw intensity levels, approximately 700 and 500, were lower than the approximate 2000 we observed in GSCs (Fig. 1B). Interestingly, patients with tumors expressing elevated levels ( $>2$-fold, $\mathrm{n}=27$ ) of CDH19 in the NCI REMBRANDT database had a significantly higher survival probability than those expressing intermediate levels ( $\mathrm{p}=0.03$, log-rank test) (Fig. 5). Although inclusion of downregulated samples provided no statistically significant increase in survival probability ( $\mathrm{p}$ $=0.07, \log$-rank test), it still offered a robust trend toward higher survival probability.

\section{Discussion}

We report the identification of $\mathrm{CDH} 19$ as a marker for minimally infiltrative GSCs through gene expression profiling, qRT-PCR, and immunoblot analysis correlated to animal tumor xenograft studies. $\mathrm{CDH} 19$ expression is restricted to cells responsible for myelination in the developing nervous systems of chickens and rats and maintains low expression in the adult human olfactory bulb and dorsal root ganglia. ${ }^{15,28}$ Our understanding of atypical Type II cadherin biology remains sparse, ${ }^{18}$ yet the little we know about $\mathrm{CDH} 19$ has prompted further investigation into its role in GBM tumorigenesis.

Crystallographic studies determined that Type II cadherins mediate homodimeric adhesion through their EC1 domain, similarly to Type I cadherins, yet structural differences suggest the inability of Type II cadherins to cross-interact with Type I cadherins, thereby conferring confined functionality within tissues..$^{18} \mathrm{CDH} 19$ is expressed developmentally in chicken Schwann cells and oligodendrocytes ${ }^{15}$ and in rat Schwann cell precursors and cranial ganglia. ${ }^{28}$ Restricted cellular expression of Type II cadherins in the developing nervous system and their functional isolation from other cadherin family members make them ideal potential markers and targets for GSC identification, isolation, and drug discovery.

CDH19-restricted expression in minimally infiltrative GSCs and its potential role in enhancing survival probability in GBM patients may not be a coincidence. GSC lines 12.1 and 22 morphologically resemble oligodendrocyte progenitor cells (OPCs) and express OPC markers such as 2', 3'-cyclic-nucleotide 3'-phosphodiesterase (CNP), a prognostically favorable immunohistochemical marker for GBM.$^{30}$ Likewise, CDH19 upregulation was prognostically favorable in NCI REMBRANDT against a subset of samples (Fig. 5). Since CDH19 expression is limited to the GSCs, pooled mRNA transcripts from a whole-tumor sample are likely diluted for this analytical 


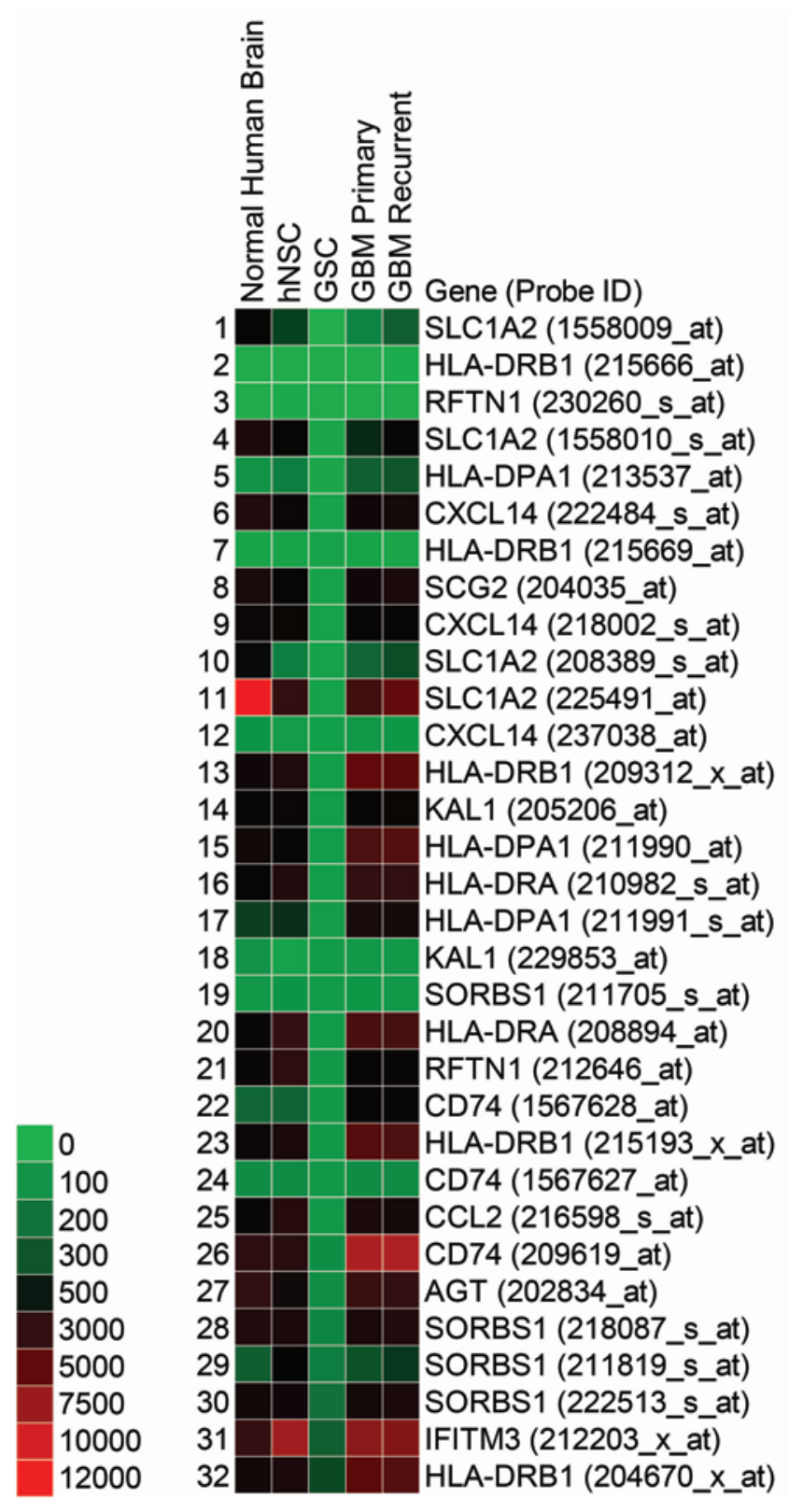

FIG. 2. Thirteen downregulated plasma membrane GSC-specific transcripts. Heat map with accompanying color key of raw intensity values from gene expression profiles. Data were filtered for plasma membrane transcripts and gated for GSC values less than 10 -fold change below all other samples. Acronyms correspond to official gene symbols found online at the NCBI. Multiple probe sets shown to demonstrate internal reproducibility.

marker. Immunohistochemical labeling seems to improve GSC resolution, as we observed with pockets of CNP expression in a clinically annotated GBM tissue microarray, ${ }^{30}$ yet CNP was not prognostically favorable in the samples of NCI REMBRANDT.

Examination of recent work published in the literature revealed a potential tumorigenic role for Type II cadherins. Transcriptional regulation of $\mathrm{CDH} 19$ and $\mathrm{CDH} 12$ was reportedly targeted by monocyte chemotactic protein 1-induced protein (MCPIP), which promoted capillary-
A

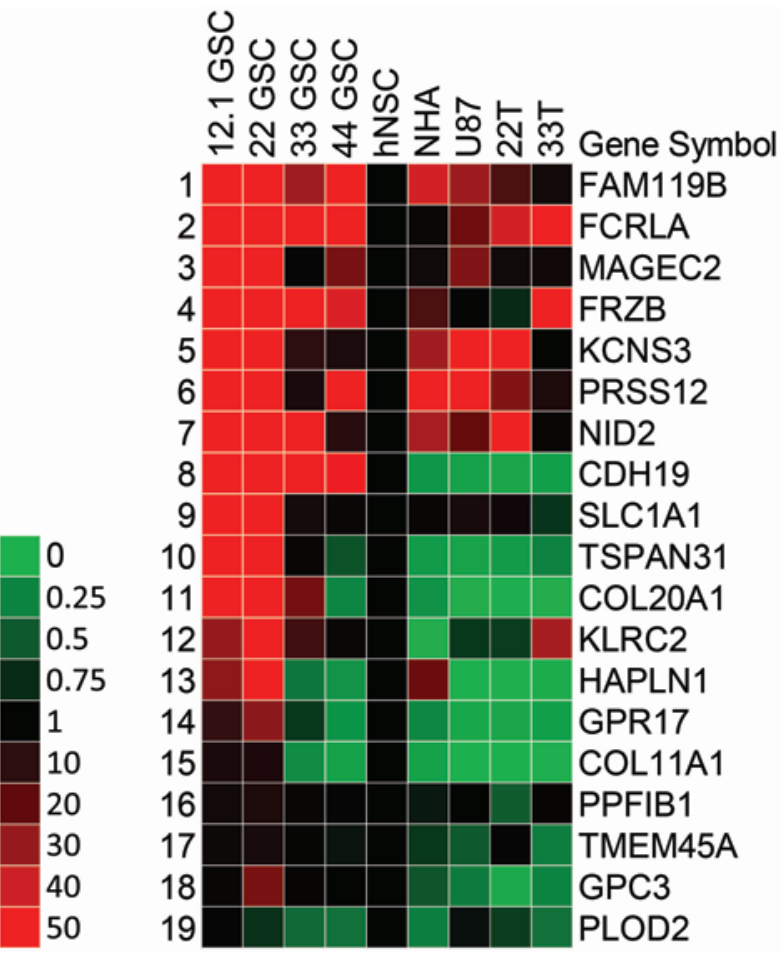

B

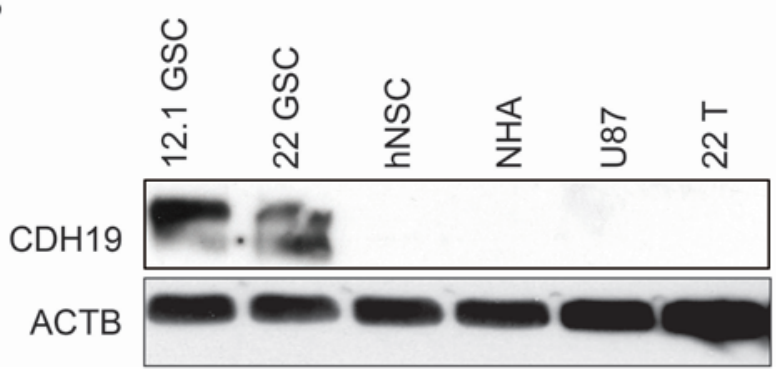

FIG. 3. Cadherin-19 is specifically expressed in minimally infiltrative GSCs. A: Heat map of qRT-PCR validation of all candidate GSC cellsurface markers. Additional GSC lines, 33 and 44, were evaluated in comparison with hNSCs, NHAs, U87 glioma cells, and patient-matched serum-cultured GBM tumor cells 22T and 33T. Data are representative of 2 experimental repetitions normalized to hNSCs and reported as a fold change value with accompanying color key. B: Western blot analysis of CDH19 specifically expressed in GSC lines 12.1 and 22. Ms-pAb-anti-CDH19 at 1:250, identified at approximately $114 \mathrm{k} \mathrm{kDa;} \mathrm{Rb-}$ pAb-ACTB at 1:2000 was used as a loading control.

like tube formation in human umbilical vein endothelial cells. ${ }^{17}$ MCPIP knockdown occurred via small interfering RNA-suppressed angiogenesis-related genes $V E G F$ and $H I F 1 A, C D H 19$, and $C D H 12$ and reduced capillary-like tube formation. Pathologically, vascular endothelial proliferation is observed in OPC-like minimally infiltrative GSC xenografts, but not in tumor xenografts from highly invasive GSCs that express abundant astrocyte progenitor cell markers. ${ }^{30}$ Perhaps $\mathrm{CDH} 19$-related upregulation of angiogenic signaling enables certain GSCs to grow into focal tumors instead of invading the normal brain parenchyma. Conversely, another Type II cadherin was found expressed as proteins in GBM and GSCs: cadherin-11 (CDH11). Knockdown of $C D H 11$ in serum-cultured U87 


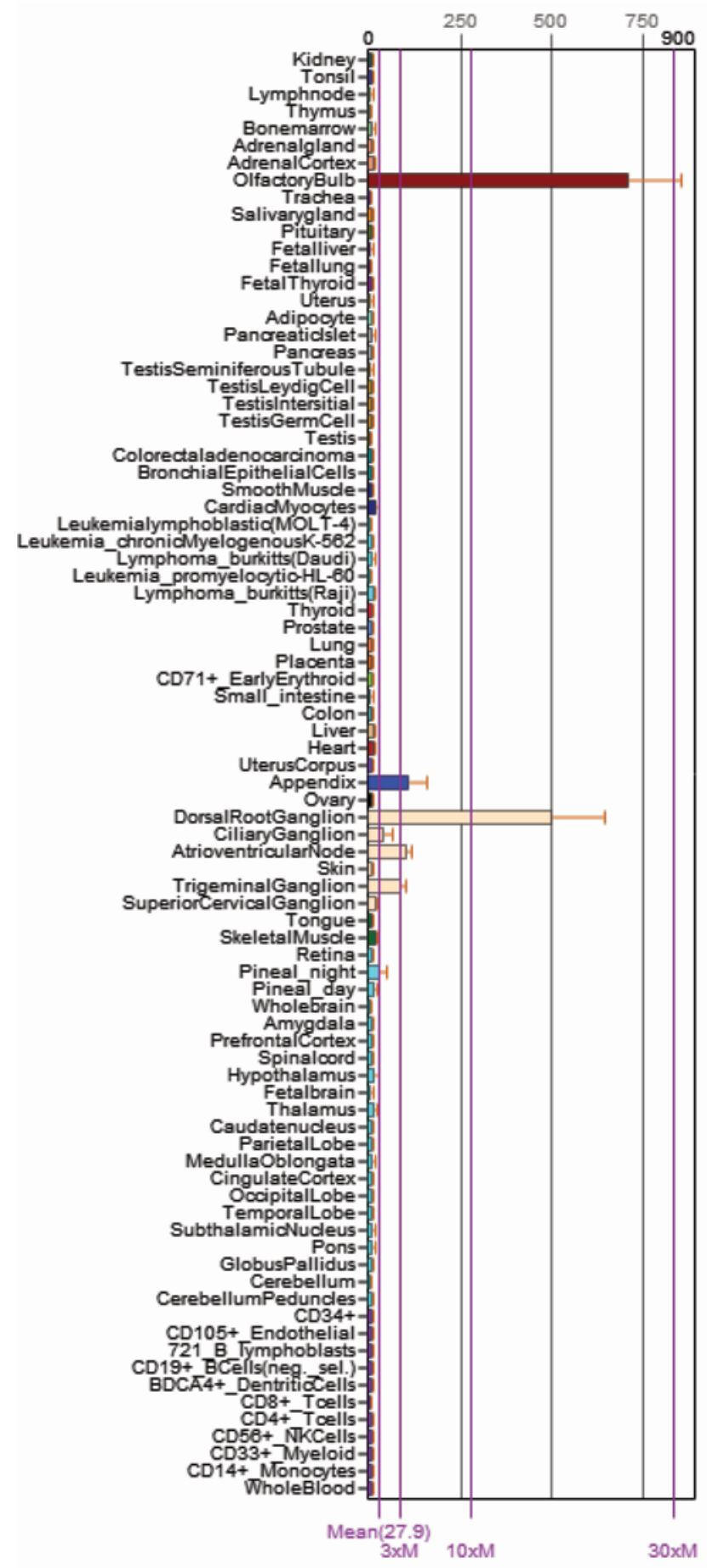

FIG. 4. Cadherin-19 transcript found in olfactory bulb and dorsal root ganglia from human tissue in BioGPS. Compiled from gene expression profiles (probe ID: 206898_at). Accessed June 5, 2013 (http://biogps. org). Figure is available in color online only.

and LN-229 GBM tumor cells reduced migration in a scratch wound assay and shortened survival in a flank xenograft model. ${ }^{13}$ Thus, each cadherin family member exerts different properties and must be studied independently to appreciate the extent of their involvement in GBM tumorigenesis.
In addition to $C D H 19$, other differentially expressed transcripts associated with cancer and oligodendrocyte biology were revealed in our studies. Glypican-3 (GPC3) transcript was upregulated in minimally infiltrative GSCs and confirmed by qRT-PCR; it was also described as a bona fide marker and drug target for hepatocellular carcinoma, ${ }^{9}$ and it was highly correlated with $\mathrm{CD} 90^{+}$liver cancer stem-like cells. ${ }^{11}$ This is the first report to establish $G P C 3$ 's relationship to GBM. Likewise, upregulation of oligodendrocyte-specific $G$ protein-coupled receptor 17 (GPR17) mRNA was validated in GSC lines 12.1 and 22, described as an intrinsic timer of myelination, ${ }^{6}$ thereby strengthening the OPC-like resemblance of these GSCs.

Three downregulated transcripts of interest were HLA Class II genes, HLA-DRB1, HLA-DPA1, and HLA-DRA, also known as major histocompatibility complex (MHC) proteins. Typically in these gene expression profiles, we observe low levels in the human brain, higher levels in GBM tumors, and downregulated expression in GSCs. Most astrocytomas are infiltrated with immune-effector cells, primarily consisting of cytotoxic $\mathrm{T}$ cells and macrophages, ${ }^{20}$ presumably due to expression of HLA/MHC Class II genes, as we have observed. This response can be activated by cytokine-based immunotherapy that targets HLA/MHC Class II molecules, ${ }^{22}$ but their eventual failure against GBMs may be due to the absence or low expression of HLA/MHC Class II molecules in GSCs that is responsible for initiating and maintaining tumor growth.

\section{Conclusions}

Our data and analysis have shown that $\mathrm{CDH} 19$ may be a suitable marker and drug target for minimally infiltrative GSCs. Its low expression in developing neuroectodermal tissue, specific upregulation in GSCs, and potential angiogenic role in tumorigenesis have prompted further development of research tools for study. Currently, no antibodies exist for live cell surface labeling of $\mathrm{CDH} 19$ that would help reveal possible associations with GSC tumor initiation. Since $C D H 19$ is typically a homodimerizing plasma membrane protein, purified $\mathrm{CDH} 19$ conjugated with corresponding visualization chemistries could potentially be used as a research tool; however, potential heteromeric cadherin-nectin complexes may complicate this analysis. ${ }^{29}$ Future experiments are planned to address CDH19's potential involvement in GSC tumor initiation, angiogenesis, migration, and proliferation. Moreover, characterization of $\mathrm{CDH} 19$ in lower-grade gliomas and oligodendrogliomas may provide more insights to glioma biology.

\section{Acknowledgments}

We thank Priya Ezhilan, Daniel Treisman, Jonathan D. Ebben, and Frank Hospod for expert technical assistance. Expert advice was generously provided by C. Svendsen, R. Vemuganti, and S. Zhang. We appreciate support from NIH (grant nos.T32GM007507, UL1RR025011, RC4AA020476, NCI HHSN261201000130C, and P30CA014520), the Wisconsin Partnership Program core grant support of the Center for Stem Cell and Regenerative Medicine, the University of Wisconsin (Graduate School, School of Medicine and Public Health and Department of Neurological Surgery), the HEADRUSH Brain Tumor Research Professorship, and Roger 


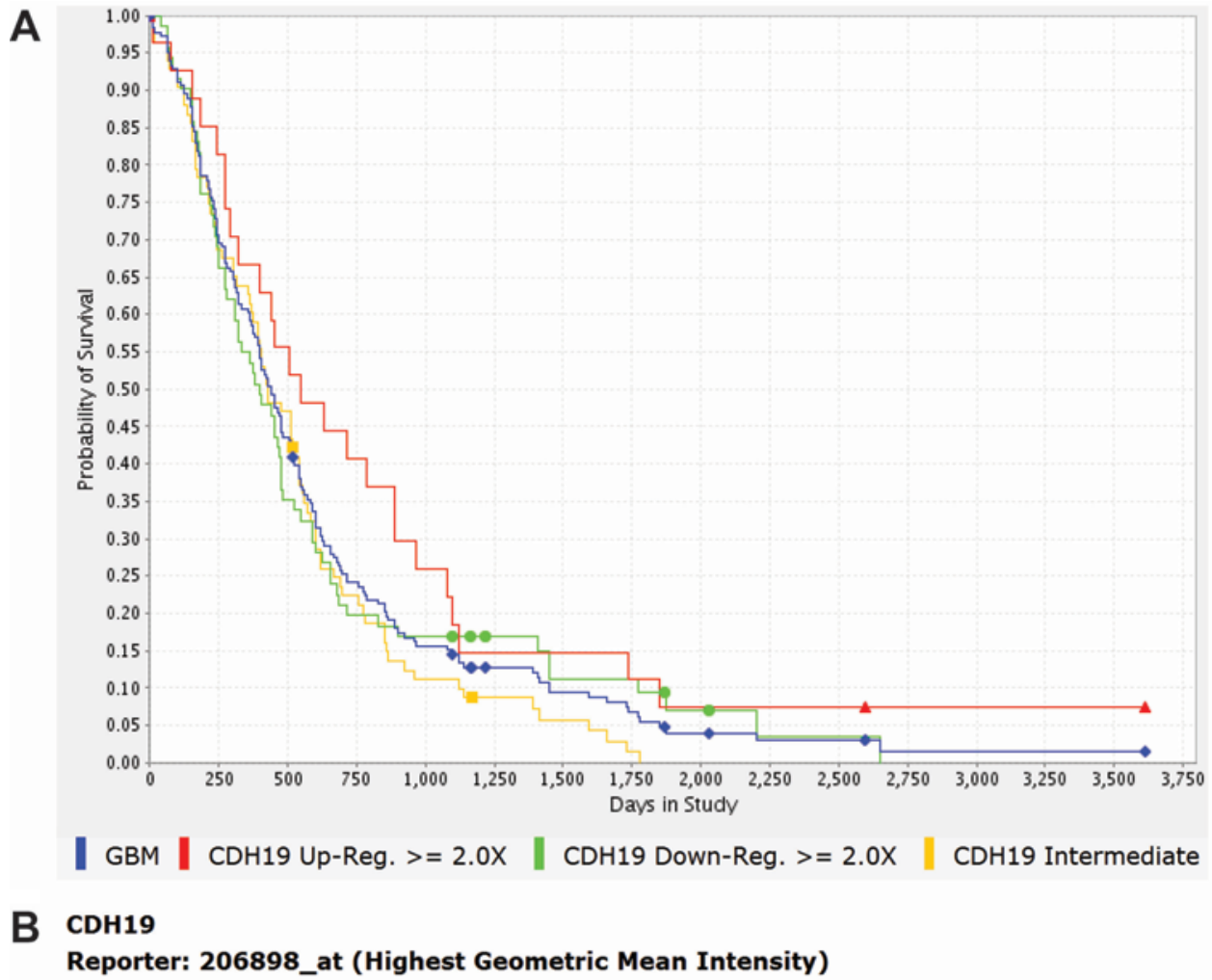

$\begin{array}{ll}\text { Number of samples in group: } & \\ \text { Up-Regulated: } & 27 \\ \text { Down-Regulated: } & 71 \\ \text { Intermediate: } & 83\end{array}$

\begin{tabular}{lc} 
Log-rank p-value(for significance of difference of survival between group of samples) \\
Up-Regulated vs. Intermediate: & 0.029276378 \\
Up-Regulated vs. Down-Regulated: & 0.2077347427 \\
Down-Regulated vs. Intermediate: & 0.4111038978 \\
\hline Up-Regulated vs. all other samples: & 0.0695107819 \\
Down-Regulated vs. all other samples: & 0.9776459531 \\
Intermediate vs. all other samples: & 0.1301745294
\end{tabular}

FIG. 5. Cadherin-19 upregulation in GBM tissue from NCI REMBRANDT showing higher survival probability. A: Kaplan-Meier survival probability curve for CDH19 (probe ID: 206898_at, highest geometrical mean intensity). Upregulation and downregulation were designated at a 2-fold difference in expression. B: Statistics generated using a log-rank test. Upregulated versus intermediate group is statistically significant for difference in survival $(p=0.03)$. Accessed June 5, 2013 (https://caintegrator.nci.nih.gov/ rembrandt). Figure is available in color online only.

Loff Memorial Fund for GBM Research. The hNSCs were a kind gift from Dr. C. Svendsen (Cedars-Sinai Medical Center, Los Angeles, California).

\section{References}

1. Al-Hajj M, Wicha MS, Benito-Hernandez A, Morrison SJ, Clarke MF: Prospective identification of tumorigenic breast cancer cells. Proc Natl Acad Sci U S A 100:3983-3988, 2003

2. Bao S, Wu Q, McLendon RE, Hao Y, Shi Q, Hjelmeland $\mathrm{AB}$, et al: Glioma stem cells promote radioresistance by preferential activation of the DNA damage response. Nature 444:756-760, 2006

3. Bleau AM, Hambardzumyan D, Ozawa T, Fomchenko EI,
Huse JT, Brennan CW, et al: PTEN/PI3K/Akt pathway regulates the side population phenotype and ABCG2 activity in glioma tumor stem-like cells. Cell Stem Cell 4:226-235, 2009

4. Bonnet D, Dick JE: Human acute myeloid leukemia is organized as a hierarchy that originates from a primitive hematopoietic cell. Nat Med 3:730-737, 1997

5. Chen R, Nishimura MC, Bumbaca SM, Kharbanda S, Forrest WF, Kasman IM, et al: A hierarchy of self-renewing tumorinitiating cell types in glioblastoma. Cancer Cell 17:362375, 2010

6. Chen Y, Wu H, Wang S, Koito H, Li J, Ye F, et al: The oligodendrocyte-specific $\mathrm{G}$ protein-coupled receptor GPR17 is a cell-intrinsic timer of myelination. Nat Neurosci 12:13981406, 2009 
7. Clark PA, Iida M, Treisman DM, Kalluri H, Ezhilan S, Zorniak M, et al: Activation of multiple ERBB family receptors mediates glioblastoma cancer stem-like cell resistance to EGFR-targeted inhibition. Neoplasia 14:420-428, 2012

8. Clark PA, Treisman DM, Ebben J, Kuo JS: Developmental signaling pathways in brain tumor-derived stem-like cells. Dev Dyn 236:3297-3308, 2007

9. Feng M, Gao W, Wang R, Chen W, Man YG, Figg WD, et al: Therapeutically targeting glypican-3 via a conformationspecific single-domain antibody in hepatocellular carcinoma. Proc Natl Acad Sci U S A 110:E1083-E1091, 2013

10. Hanahan D, Weinberg RA: Hallmarks of cancer: the next generation. Cell 144:646-674, 2011

11. Ho DW, Yang ZF, Yi K, Lam CT, Ng MN, Yu WC, et al: Gene expression profiling of liver cancer stem cells by RNAsequencing. PLoS ONE 7:e37159, 2012

12. Ignatova TN, Kukekov VG, Laywell ED, Suslov ON, Vrionis FD, Steindler DA: Human cortical glial tumors contain neural stem-like cells expressing astroglial and neuronal markers in vitro. Glia 39:193-206, 2002

13. Kaur H, Phillips-Mason PJ, Burden-Gulley SM, KerstetterFogle AE, Basilion JP, Sloan AE, et al: Cadherin-11, a marker of the mesenchymal phenotype, regulates glioblastoma cell migration and survival in vivo. Mol Cancer Res 10:293-304, 2012

14. Lee J, Kotliarova S, Kotliarov Y, Li A, Su Q, Donin NM, et al: Tumor stem cells derived from glioblastomas cultured in bFGF and EGF more closely mirror the phenotype and genotype of primary tumors than do serum-cultured cell lines. Cancer Cell 9:391-403, 2006

15. Lin J, Luo J, Redies C: Cadherin-19 expression is restricted to myelin-forming cells in the chicken embryo. Neuroscience 165:168-178, 2010

16. Liu G, Yuan X, Zeng Z, Tunici P, Ng H, Abdulkadir IR, et al: Analysis of gene expression and chemoresistance of CD133+ cancer stem cells in glioblastoma. Mol Cancer 5:67, 2006

17. Niu J, Azfer A, Zhelyabovska O, Fatma S, Kolattukudy PE: Monocyte chemotactic protein (MCP)-1 promotes angiogenesis via a novel transcription factor, MCP-1-induced protein (MCPIP). J Biol Chem 283:14542-14551, 2008

18. Patel SD, Ciatto C, Chen CP, Bahna F, Rajebhosale M, Arkus $\mathrm{N}$, et al: Type II cadherin ectodomain structures: implications for classical cadherin specificity. Cell 124:1255-1268, 2006

19. Patru C, Romao L, Varlet P, Coulombel L, Raponi E, Cadusseau J, et al: CD133, CD15/SSEA-1, CD34 or side populations do not resume tumor-initiating properties of long-term cultured cancer stem cells from human malignant glio-neuronal tumors. BMC Cancer 10:66, 2010

20. Plautz GE, Miller DW, Barnett GH, Stevens GH, Maffett S, Kim J, et al: T cell adoptive immunotherapy of newly diagnosed gliomas. Clin Cancer Res 6:2209-2218, 2000

21. Reya T, Morrison SJ, Clarke MF, Weissman IL: Stem cells, cancer, and cancer stem cells. Nature 414:105-111, 2001

22. Rosenberg SA: Progress in human tumour immunology and immunotherapy. Nature 411:380-384, 2001

23. Singh SK, Clarke ID, Terasaki M, Bonn VE, Hawkins C,
Squire J, et al: Identification of a cancer stem cell in human brain tumors. Cancer Res 63:5821-5828, 2003

24. Singh SK, Hawkins C, Clarke ID, Squire JA, Bayani J, Hide $\mathrm{T}$, et al: Identification of human brain tumour initiating cells. Nature 432:396-401, 2004

25. Son MJ, Woolard K, Nam DH, Lee J, Fine HA: SSEA-1 is an enrichment marker for tumor-initiating cells in human glioblastoma. Cell Stem Cell 4:440-452, 2009

26. Stupp R, Mason WP, van den Bent MJ, Weller M, Fisher $\mathrm{B}$, Taphoorn MJ, et al: Radiotherapy plus concomitant and adjuvant temozolomide for glioblastoma. N Engl J Med 352:987-996, 2005

27. Svendsen CN, ter Borg MG, Armstrong RJ, Rosser AE, Chandran S, Ostenfeld T, et al: A new method for the rapid and long term growth of human neural precursor cells. J Neurosci Methods 85:141-152, 1998

28. Takahashi M, Osumi N: Identification of a novel type II classical cadherin: rat cadherin19 is expressed in the cranial ganglia and Schwann cell precursors during development. Dev Dyn 232:200-208, 2005

29. Tanaka Y, Nakanishi H, Kakunaga S, Okabe N, Kawakatsu T, Shimizu K, et al: Role of nectin in formation of E-cadherinbased adherens junctions in keratinocytes: analysis with the N-cadherin dominant negative mutant. Mol Biol Cell 14:1597-1609, 2003

30. Zorniak M, Clark PA, Leeper HE, Tipping MD, Francis DM, Kozak KR, et al: Differential expression of 2',3'-cyclicnucleotide $3^{\prime}$-phosphodiesterase and neural lineage markers correlate with glioblastoma xenograft infiltration and patient survival. Clin Cancer Res 18:3628-3636, 2012

\section{Author Contributions}

Conception and design: all authors. Acquisition of data: Zorniak, Clark. Analysis and interpretation of data: all authors. Drafting the article: Zorniak. Critically revising the article: all authors. Reviewed submitted version of manuscript: all authors. Approved the final version of the manuscript on behalf of all authors: Kuo. Statistical analysis: Zorniak. Administrative/technical/material support: Kuo, Clark. Study supervision: all authors.

\section{Supplemental Information \\ Database Repository and Accession Numbers}

GSC lines 12.1 (NCBI GEO accession no. GSM1253303) and 22 GSC (NCBI GEO accession no. GSM1253304); hNSCs M031 CTX (NCBI GEO accession nos. GSM458064 and GSM458065); Normal Human Brain, GBM Primary, GBM Recurrent (NCI REMBRANDT; Table 1).

\section{Correspondence}

John S. Kuo, Department of Neurological Surgery, University of Wisconsin School of Medicine and Public Health, Box 8660 Clinical Science Center, 600 Highland Ave., Madison, WI 53792 8660.email: j.kuo@neurosurgery.wisc.edu. 